\title{
Universal portfolio analysis of Malaysia's stocks for different durations
}

\author{
Sook Theng Pang ${ }^{1 *}$, and How Hui Liew ${ }^{1}$ \\ ${ }^{1}$ Department of Mathematical and Actuarial Sciences, Universiti Tunku Abdul Rahman, Jalan Sungai \\ Long, Bandar Sungai Long, Cheras, 43000 Kajang, Malaysia
}

\begin{abstract}
In this research, four proposed finite order universal portfolios were used to study Malaysia's stock market comprehensively and the constant rebalanced portfolio (CRP) was used as a benchmark for comparison. The empirical performance of the four universal portfolio strategies was analysed experimentally concerning 95 stocks from different categories in Kuala Lumpur Stock Exchange (KLSE) from 1 January 2000 to 31 December 2015. Combinations of three stocks data from the selected 95 stocks are used for study for short-term (1-year duration), middle-term (4-years and 8-years durations) and long-term (12-years and 16-years durations). The empirical results showed that the performances of the proposed universal strategies are outperform CRP in 1 year and 4 years durations, but did poorly in 8-years, 12-years and 16-years durations. Therefore, these four UP strategies are empirically considered to be good investment strategies in the short-term.
\end{abstract}

\section{Introduction}

The goal of this research is to study Malaysia's stock market using four finite order universal portfolios (UP) comprehensively and to use the constant rebalanced portfolio (CRP) as a benchmark. Finite order universal portfolio depends only on the positive moments of the generating probability distribution. In this research, the formulation of the

-weighted universal portfolio, Cover-Ordentlich universal portfolio [1] and order universal portfolio [2] were simplified using probability theory and they are further extended to stochastic process based universal portfolio. The order universal portfolio has better speed and memory performance as well as being easier to implement in highlevel programming language compare to the original Cover-Ordentlich universal portfolio.

In Tan et al. [3], a study of empirical performance of the universal portfolio generated by certain reciprocal functions of the price relatives is presented. Raymond et al. [4] study the portfolio diversification strategy namely active and passive portfolio investment strategy in Malaysian stock market. The overall outcomes showed that the active portfolio strategy outperforms the passive portfolio strategy.

This research was conducted with 95 stocks data from KLSE (Kuala Lumpur Stock Exchange) for the duration from 1 January 2000 to 31 December 2015. The four finite

*Corresponding author: pangst@utar.edu.my 
order universal portfolios (UPs) used in this research were multinomial UP, multivariate normal UP, Brownian-motion generated UP and Ornstein-Uhlenbeck OU process generated UP. This research limit to order one UP and 3 stocks, $m=3$. Using the basic probability theory, the four proposed finite order universal portfolio are formalised.

\section{Order one universal portfolio}

\subsection{General algorithm}

Consider an $m$-stock market. Let $x_{n}=\left(x_{n, i}\right)$ be the stock-price-relative vector on the $n^{\text {th }}$ trading day, where $x_{n, i}$ denotes the stock-price relative of stock $i$ on day $n$, which is defined to be the ratio of the closing price to its opening price on day $n$, for $i=1, \ldots ., m$. We assume that $x_{n, i} \geq 0$, for all $i=1,2, \cdots, m$ and $n=1,2, \cdots$. The portfolio vector $b_{n}=\left(b_{n, i}\right)$ on day $n$ is the investment strategy used on day $n$ when $b_{n, i}$ is the proportion of the current wealth invested on stock $i$ for $i=1,2, \cdots, m$ with $0 \quad b_{n, i} \quad 1$ and $\sum_{i=1}^{m} b_{n, i}=1$.

Cover [5] introduced an active trading strategy which involves a sequence of universal portfolio vector $\mathbf{b}_{n}=\left(b_{n, 1}, \cdots, b_{n, m}\right)$, which are associated with the $n$ trading days. Here, the value $b_{n, j}, i=1,2, \cdots, m$ is the proportion of the wealth of the asset $i$ on the $n$th day and $\sum_{i=1}^{m} b_{n, i}=1$. The wealth at the end of $n$th trading day $S_{n}$ is given by

$$
S_{n}=S_{0} \prod_{j=1}^{n} \mathbf{b}_{j}^{t} \mathbf{x}_{j}
$$

A constant rebalanced portfolio $(\mathrm{CRP})$ is a portfolio $\mathrm{b}_{j}=(\mathrm{b})$ that is constant over the trading days and the wealth at the end of $n$th trading day is

$$
S_{n}=S_{0} \prod_{j=1}^{n} \mathbf{b}^{t} \mathbf{x}_{j}
$$

The following are the wealth and update scheme used in Cover \& Ordentlich [1].

$$
\begin{aligned}
& \mathrm{b}_{n+1}=\frac{\int_{\Delta_{m}} \mathrm{~b} S_{n}(\mathrm{~b}) d \mu(\mathrm{b})}{\int_{\Delta_{m}} S_{n}(\mathrm{~b}) d \mu(\mathbf{b})} \\
& S_{n}=\int_{\Delta_{\mathbf{m}}} S_{n}(\mathbf{b}) d \mu(\mathbf{b})
\end{aligned}
$$

where $S_{n}(\mathrm{~b})=\prod_{j=1}^{n} \mathrm{~b}_{j}^{t} \mathrm{x}_{j}$.

Let $\mathrm{Y}=\left(Y_{1}, \cdots, Y_{m}\right)$ be an $m$-dimensional random vector such that $0 \quad Y_{i} \quad 1$ and $Y_{1}+$ $\cdots+Y_{m}=1$. By using the notation of expected value [6] and replacing the random vector in (3) with Y, it can be written as 


$$
\mathrm{b}_{n+1}=\frac{E\left[Y \prod_{j=1}^{n} \mathrm{Y}^{t} \mathrm{x}_{j}\right]}{E\left[\prod_{j=1}^{n} Y^{t} \mathrm{x}_{j}\right]}=\frac{E\left[Y\left(Y^{t} x_{n}\right)\left(Y^{t} x_{n-1}\right) \cdots\left(Y^{t} x_{1}\right)\right]}{E\left[\left(Y^{t} x_{n}\right)\left(Y^{t} x_{n-1}\right) \cdots\left(Y^{t} x_{1}\right)\right]}
$$

The $k$ component of the vector $\mathrm{b}_{n+1}$ is

$$
\mathrm{b}_{n+1}=\frac{E\left[Y_{k}\left(Y^{t} x_{n}\right)\left(Y^{t} x_{n-1}\right) \cdots\left(Y^{t} x_{1}\right)\right]}{E\left[\left(Y^{t} x_{n}\right)\left(Y^{t} x_{n-1}\right) \cdots\left(Y^{t} x_{1}\right)\right]}
$$

where $k=1,2 ., \ldots, m$.

Tan [2] introduced a variation of Cover's universal portfolio to deal with inefficiency of Cover's universal portfolio, call Probability-Based Order- Universal Portfolio. In contrast to Cover, Tan [2] introduced a random vector such that $Y_{i}>0$ and define

$$
\mathbf{b}_{n+1}=\frac{E\left[Y\left(Y^{t} x_{n}\right)\left(Y^{t} x_{n-1}\right) \cdots\left(Y^{t} x_{n-v+1}\right)\right]}{E\left[\left(Y_{1}+Y_{2}+\cdots+Y_{m}\right)\left(Y^{t} x_{n}\right)\left(Y^{t} x_{n-1}\right) \cdots\left(Y^{t} x_{n-v+1}\right)\right]}
$$

where the $k$ component has the form

$$
\mathrm{b}_{n+1}=\frac{E\left[Y_{k}\left(Y^{t} x_{n}\right)\left(Y^{t} x_{n-1}\right) \cdots\left(Y^{t} x_{n-v+1}\right)\right]}{E\left[\left(Y_{1}+Y_{2}+\cdots+Y_{m}\right)\left(Y^{t} x_{n}\right)\left(Y^{t} x_{n-1}\right) \cdots\left(Y^{t} x_{n-v+1}\right)\right]}
$$

with these notations, the wealth formula (1) can be expressed as

$$
S_{n}=\left(\mathbf{b}_{n}^{t} \mathbf{x}_{n}\right)\left(\mathbf{b}_{n-1}^{t} \mathbf{x}_{n-1}\right) \cdots\left(\mathbf{b}_{n-v+1}^{t} \mathbf{x}_{n-v+1}\right) S_{n-v}
$$

when $=1$,

$$
S_{n}=\left(\mathbf{b}_{n}^{t} \mathbf{x}_{n}\right) S_{n-v}
$$

The algorithm for computing (8) can be defined by expanding the numerator as follows:

$$
\begin{aligned}
& E\left[Y_{k}\left(\mathrm{Y}^{t} \mathbf{x}_{n}\right) \cdots\left(\mathrm{Y}^{t} \mathbf{x}_{n-v+1}\right)\right] \\
= & E\left[Y_{k} \prod_{j=n-v+1}^{m}\left(\sum_{i=1}^{m} Y_{i} x_{j, i}\right)\right] \\
= & E\left[Y_{k} \sum_{i_{1}=1}^{m} \sum_{i_{2}=1}^{m} \sum_{i_{3}=1}^{m} \cdots \sum_{i_{v}=1}^{m} Y_{i_{1}} \cdots Y_{i_{v}} x_{n, i_{1}} \cdots x_{n-v+1, i_{v}}\right] \\
= & \sum_{i_{1}=1}^{m} \sum_{i_{2}=1}^{m} \sum_{i_{3}=1}^{m} \cdots \sum_{i_{v}=1}^{m} x_{n, i_{1}} \cdots x_{n-v+1, i_{v}} \sum_{j=1}^{m} E\left[Y_{k} Y_{i_{1}} \cdots Y_{i_{v}}\right]
\end{aligned}
$$


The expansion of denominator of $(8)$ is shown below:

$$
\begin{aligned}
& E\left[\left(Y_{1}+\cdots+Y_{m}\right)\left(\mathrm{Y}^{t} \mathbf{x}_{n}\right) \cdots\left(\mathrm{Y}^{t} \mathbf{x}_{n-v+1}\right)\right] \\
& =\sum_{j=1}^{m} E\left[Y_{j}\left(\mathrm{Y}^{t} \mathbf{x}_{m}\right) \cdots\left(\mathrm{Y}^{t} \mathbf{x}_{n-v+1}\right)\right] \\
& =\sum_{j=1}^{m} \sum_{i} \sum_{1}=1 \sum_{i_{2}=1}^{m} \cdots \sum_{i_{v}=1}^{m} x_{n, i_{1}} \cdots x_{n-v+1, i_{v}} E\left[Y_{j} Y_{i_{1}} \cdots Y_{i_{v}}\right] \\
& =\sum_{i_{1}=1}^{m} \sum_{i_{2}}^{m} \sum_{i}^{m} \cdots \sum_{i_{v}=1}^{m} x_{n, i_{1}} \cdots x_{n-v+1, i_{v}} \sum_{j=1}^{m} E\left[Y_{j} Y_{i_{1}} \cdots Y_{i_{v}}\right]
\end{aligned}
$$

When $=1,(8)$ becomes

$$
b_{n+1, k}=\frac{\sum_{i=1}^{m} x_{n, i} E\left[Y_{k} Y_{i}\right]}{\sum_{i=1}^{m} x_{n, i}\left(\sum_{j=1}^{m} E\left[Y_{j} Y_{i}\right]\right)} .
$$

The algorithms for the four proposed universal portfolios were explored in Pang et al. [7, 8]. The best performance parameters for the four strategies are studied in Pang et al. [7, 8].

\section{Results and discussion}

\subsection{Performance analysis of four universal portfolio strategies against CRP}

The performance of the four UP strategies is analysed for the empirically best performing parameters found in Pang et al. [7, 8]. Numerical experiment for the four UP strategies is studied concerning 95 stocks from different categories in Kuala Lumpur Stock Exchange from 1 January 2000 to 31 December 2015. Every 3 stocks data generated by combinations of the selected 95 stocks are used for study for short-term (1-year duration), middle-term (4-years and 8-years duration) and long-term (12-years and 16-years duration). The experiment will be limited to order one universal portfolio and CRP as a benchmark.

The descriptive statistics of the ratios of the return of our four strategies against the return of the CRP wealth are summarised in Table 1 to Table 5.

Table 1. Short term duration data analysis (1 year duration).

\begin{tabular}{|l|c|l|c|l|}
\hline Portfolio Strategies & Min & Median & Mean & Max \\
\hline Multinomial UP & -2.9900 & 0.0720 & 0.1134 & 3.1460 \\
\hline Multivariate Normal UP & -2.9540 & 0.0672 & 0.1074 & 3.0750 \\
\hline Brownian Motion UP & -2.8480 & 0.0578 & 0.0939 & 2.8910 \\
\hline OU Process UP & -3.0830 & 0.0666 & 0.1075 & 3.1761 \\
\hline
\end{tabular}

Table 2. Middle term duration data analysis (4 years duration).

\begin{tabular}{|l|l|l|l|l|}
\hline Portfolio Strategies & \multicolumn{1}{|c|}{ Min } & Median & Mean & Max \\
\hline Multinomial UP & -3.6420 & 0.0653 & 0.1245 & 4.3460 \\
\hline Multivariate Normal UP & -3.5830 & 0.0579 & 0.1161 & 4.2520 \\
\hline Brownian Motion UP & -3.4300 & 0.0446 & 0.0980 & 4.0050 \\
\hline OU Process UP & -3.7330 & 0.0552 & 0.1143 & 4.3960 \\
\hline
\end{tabular}


From Tables $1 \& 2$, the median and mean are positive values for the four UPs, this indicate that the performance of the four proposed UP strategies are better than CRP.

Table 3. Middle term duration data analysis (8 years duration).

\begin{tabular}{|l|c|l|l|l|}
\hline Portfolio Strategies & \multicolumn{1}{|c|}{ Min } & Median & Mean & Max \\
\hline Multinomial UP & -8.4310 & -0.0226 & -0.1555 & 5.8400 \\
\hline Multivariate Normal UP & -9.0200 & -0.0247 & -0.1704 & 6.1520 \\
\hline Brownian Motion UP & -9.3050 & -0.0256 & -0.1818 & 6.2600 \\
\hline OU Process UP & -9.3500 & -0.0327 & -0.1840 & 6.3660 \\
\hline
\end{tabular}

Table 4. Long term duration data analysis (12 years duration).

\begin{tabular}{|l|c|c|c|c|}
\hline Portfolio Strategies & Min & Median & Mean & Max \\
\hline Multinomial UP & -inf & 0 & Inf & 16 \\
\hline Multivariate Normal UP & -inf & 0 & Inf & 15 \\
\hline Brownian Motion UP & -inf & 0 & Inf & 16 \\
\hline OU Process UP & -inf & 0 & inf & 16 \\
\hline
\end{tabular}

Table 5. Long term duration data analysis (16 years duration).

\begin{tabular}{|l|c|c|c|c|}
\hline Portfolio Strategies & Min & Median & Mean & Max \\
\hline Multinomial UP & -inf & 0 & Inf & 40 \\
\hline Multivariate Normal UP & -inf & 0 & Inf & 38 \\
\hline Brownian Motion UP & -inf & 0 & Inf & 39 \\
\hline OU Process UP & -inf & 0 & Inf & 40 \\
\hline
\end{tabular}

Tables 3, $4 \& 5$ showed that the CRP performed better than the four proposed UP strategies. There are some extreme values showed under mean, minimum value and maximum value. Therefore, the summary values obtained in long term period only meaningful if we eliminate the outliers. The maximum values obtained for four proposed strategies are much larger than CRP.

\section{Conclusion}

The empirical results showed that the performances are better than CRP for a 1-year duration and a 4-years duration. However, the four UP strategies did poorly for 8-years, 12-years, and 16-years durations. Therefore, these four UP strategies are empirically considered to be good investment strategies in the short-term. A further study for the cause of the poor performance of the proposed four UP strategies in medium and long terms will be carried out in the future.

So far, the research of universal portfolios in Malaysia was focused in determining the best performing parameters for $m$ stocks [3], [7, 8]. The result of this study showed that the four UP are performed well compared to CRP for short term, As a conclusion, this study indicates that the order one universal portfolios may help to promote active trading for Malaysia stock market in short term.

\section{References}

1. T.M. Cover, E. Ordentlich, IEEE Trans. Inform. Theory 42, 348 - 363 (1996) 
2. C.P. Tan, Performance bounds for the distribution generated universal portfolio, in Proceeding of the $59^{\text {th }}$ World Statistics Congress of the International Statistical institute, 5327 - 5332 (2013)

3. C.P. Tan, K.S. Kuang, S.W. Phoon, IOSR-JM 11, 54 - 61 (2015)

4. L. Raymond, J.Y. Chia, Capital Market Review 24(1), 38 - 67 (2016)

5. T.M. Cover, Math. Financ. 1, 1 - 29 (1991)

6. D.D. Wackerly, W. III. Mendenhall, R.L. Scheaffer, Mathematical Statistics with application (Brooks/Cole, Canada, 279 - 285, 2000)

7. S.T. Pang, H.H. Liew, Y.F. Chang, Performance of finite order distributed generated universal portfolio, The $4^{\text {th }}$ International Conference of Mathematical Sciences, UKMM AIP Conference Proceedings 1830, 020059 (2017)

8. S.T. Pang, H.H. Liew, Y.F. Chang, MJMS, UPM 13, 157 - 171 (2019) 